\title{
Fronto-Striatal Glutamate in Autism Spectrum Disorder and Obsessive Compulsive Disorder
}

\author{
Jilly Naaijen*,1, Marcel P Zwiers', Houshang Amiri ${ }^{1,2}$, Steven CR Williams ${ }^{3}$, Sarah Durston ${ }^{4}$, \\ Bob Oranje ${ }^{4}$, Daniel Brandeis ${ }^{5,6,7,8}$, Regina Boecker-Schlier ${ }^{5}$, Matthias Ruf ${ }^{9}$, Isabella Wolf ${ }^{5,9}$, \\ Tobias Banaschewski ${ }^{5}$, Jeffrey C Glennon', Barbara Franke ${ }^{10,11}$, Jan K Buitelaar ${ }^{1,12}$ and David J Lythgoe ${ }^{3}$ \\ for The TACTICS Consortium ${ }^{13}$
}

\begin{abstract}
'Department of Cognitive Neuroscience, Donders Institute for Brain, Cognition and Behavior, Radboud University Medical Center, Nijmegen, The Netherlands; ${ }^{2}$ Neuroscience Research Center, Institute of Neuropharmacology, Kerman University of Medical Sciences, Kerman, Iran; ${ }^{3}$ Department of Neuroimaging, King's College London, Institute of Psychiatry, Psychology, and Neuroscience, London, UK; ${ }^{4}$ NICHE Lab, Department of Psychiatry, Brain Center Rudolf Magnus, University Medical Center Utrecht, Utrecht, The Netherlands; ${ }^{5}$ Department of Child and Adolescent Psychiatry and Psychotherapy, Central Institute of Mental Health, Medical Faculty, Heidelberg University, Mannheim, Germany; ${ }^{6}$ Department of Child and Adolescent Psychiatry and Psychotherapy, Psychiatric Hospital, University of Zurich, Zurich, Switzerland; ${ }^{7}$ Center for Integrative Human Physiology, University of Zurich, Zurich, Switzerland; ${ }^{8}$ Neuroscience Center Zurich, University of Zurich, ETH Zurich, Zurich, Switzerland; ${ }^{9}$ Department of Neuroimaging, Central Institute of Mental Health, Medical Faculty, Heidelberg University, Mannheim, Germany; ${ }^{10}$ Department of Human Genetics, Donders Institute of Brain, Cognition and Behavior, Radboud University Medical Center, Nijmegen, The Netherlands; "'Department of Psychiatry, Donders Institute of Brain, Cognition and Behavior, Radboud University Medical Center, Nijmegen, The Netherlands; ${ }^{12}$ Karakter Child and Adolescent Psychiatry University Center, Nijmegen, The Netherlands
\end{abstract}

\begin{abstract}
Autism spectrum disorders (ASDs) and obsessive compulsive disorder (OCD) are often comorbid with the overlap based on compulsive behaviors. Although previous studies suggest glutamatergic deficits in fronto-striatal brain areas in both disorders, this is the first study to directly compare the glutamate concentrations across the two disorders with those in healthy control participants using both categorical and dimensional approaches. In the current multi-center study (four centers), we used proton magnetic resonance spectroscopy in $5 \mathrm{I}$ children with ASD, 29 with OCD, and 53 healthy controls (aged 8-13 years) to investigate glutamate (Glu) concentrations in two regions of the fronto-striatal circuit: midline anterior cingulate cortex (ACC) and left dorsal striatum. Spectra were processed with Linear Combination Model. Group comparisons were performed with one-way analyses of variance including sex, medication use, and scanner site as covariates. In addition, a dimensional analysis was performed, linking glutamate with a continuous measure of compulsivity across disorders. There was a main group effect for ACC glutamate $(p=0.019)$. Contrast analyses showed increased glutamate both in children with ASD and OCD compared with controls $(p=0.007)$, but no differences between the two disorders $(p=0.770)$. Dimensional analyses revealed a positive correlation between compulsive behavior (measured with the Repetitive Behavior Scale) and ACC glutamate (rho $=0.24, p=0.03$ ). These findings were robust across sites. No differences were found in the striatum. The current findings confirm overlap between ASD and OCD in terms of glutamate involvement. Glutamate concentration in ACC seems to be associated with the severity of compulsive behavior. Neuropsychopharmacology (2017) 42, 2456-2465; doi: I0.1038/npp.2016.260; published online I4 December 20I6
\end{abstract}

\section{INTRODUCTION}

Autism spectrum disorders (ASDs) are characterized by deficits in reciprocal social interaction, impairments in communication, and by restricted, repetitive, and stereotyped patterns of behavior, and interests. Similarly, obsessive compulsive disorder (OCD) is characterized by repetitive thoughts, impulses, or images (obsessions), and repetitive

*Correspondence: J Naaijen, MSc, Department of Cognitive Neuroscience, Donders Institute for Brain, Cognition and Behavior, Radboud University Medical Center, P.O. Box 9101, Nijmegen 6500 HB, The Netherlands, Tel: +3I 24 36I0986, E-mail: j.naaijen@donders.ru.nl

${ }^{13}$ Members of The TACTICS Consortium are listed in the Acknowledgments.

Received 2 October 2016; revised II November 2016; accepted I4 November 2016; accepted article preview online 2I November 2016 behaviors or mental acts (compulsions) that cause marked distress and/or impairment of functioning (American Psychiatric Association, 2000). Despite the diagnostic differences between these disorders, there is also strong clinical overlap and comorbidity. Both ASD and OCD show repetitive behaviors among their core features, and comparison of their symptoms has shown more overlap than differences (Anholt et al, 2010). A recent longitudinal investigation showed that individuals diagnosed with ASD were at twofold increased risk of developing OCD, and vice versa, that OCD patients were at a fourfold increased risk of having ASD, compared with people with no prior diagnosis (Meier et al, 2015).

Compulsivity is a cross-disorder trait that appears to overlap between ASD and OCD. Compulsivity is defined as the repetitive and irresistible urge to perform certain 
behaviors, the experience of loss of voluntary control over this urge, the diminished ability to delay or inhibit thoughts and behaviors, and the tendency to perform repetitive acts in a habitual or stereotyped manner (Chamberlain and Menzies, 2009). Although the psychological mechanism underlying the compulsive behavior is different for ASD (self-soothing) and OCD (reducing stress), poor behavioral inhibition has been linked to both disorders (Robbins et al, 2012). The prefrontal cortex (PFC) acts in coordination with the striatum to implement the control of behavior.

It has therefore been suggested that changes in this frontostriatal circuit may be involved in the compulsivity seen in ASD and OCD (Fuccillo, 2016; Voon et al, 2014). According to neuroanatomical models, the fronto-striatal circuit includes a compulsivity route, in which the dorsal striatum drives the compulsive behavior, while the PFC exerts control over it (Alexander et al, 1986). A recent network analysis provided evidence that the overlap between ASD and OCD was mainly related to repetitive behaviors (Ruzzano et al, 2015), which have been linked to deficits in executive functioning of the fronto-striatal circuit (Thakkar et al, 2008).

An important neurotransmitter involved in the functioning of fronto-striatal circuits is glutamate. Glutamate is the most abundant excitatory neurotransmitter in the human central nervous system, involved in processes such as synaptic transmission, plasticity and long-term potentiation (Pittenger et al, 2011). Proton magnetic resonance spectroscopy (MRS) is a non-invasive method allowing for in vivo quantification of several neurometabolites, including glutamate (Glu). Although MRS is not suitable for distinguishing glial, metabolic, and neurotransmitter glutamate, there is evidence suggesting that the glutamate-glutamine cycle, responsible for glutamate supply as a neurotransmitter, accounts for $\sim 80 \%$ of the glutamate trafficking in the brain (Hyder et al, 2013). Previous MRS studies in ASD and OCD have shown alterations in glutamate in fronto-striatal circuits (for an overview, see Naaijen et al, 2015). However, many of these previous studies were based on rather small sample sizes and used low field strengths that did not allow accurate separation of Glu from glutamine (Gln); those studies therefore reported the combined Glu+Gln signal (the so-called Glx), which is less informative. Although studies differed greatly, many of them found increased glutamate levels in prefrontal brain areas in ASD compared with controls. This increase was age-dependent, and present in children, whereas a reversed pattern was observed in adults (Naaijen et al, 2015). Striatal findings have been more inconsistent, but so far increased striatal glutamate levels have been found in neurodevelopmental disorders (including ASD and OCD) compared with controls as well (ie, Hassan et al, 2013; Rosenberg et al, 2000).

In the present study, we investigated whether glutamatergic concentrations in the fronto-striatal circuit differed in children with compulsivity disorders relative to healthy controls. We specifically focused on the age range between 8 and 13 years, as the transition from childhood to adolescence begins in this period, in which proper functioning of the fronto-striatal circuit is essential (Somerville and Casey, 2010). In addition, as a dimensional cross-disorder comparison, we investigated whether glutamate concentrations were associated with a continuous measure of compulsivity. Because prefrontal glutamate has been associated with anxiety before (Klumpp et al, 2011; Modi et al, 2014), we also included an association with a continuous measure for anxiety to investigate whether possible relations are compulsivity specific. We focused on two regions of interest, the midline anterior cingulate cortex (ACC) and the left dorsal striatum (covering caudate and putamen) to be able to compare our results with several previous studies performed in ASD and OCD. We expected ASD patients to show higher glutamate levels compared with healthy controls in both regions. Although to date the findings in OCD have been less robust, we expected changes to be comparable to the ASD group, because of the overlap of the two disorders in compulsive behavior.

\section{MATERIALS AND METHODS}

\section{Participants}

We included 68 pediatric participants with ASD, 34 with OCD, and 61 healthy controls. Participants (aged 8-13 years) were recruited across four sites in Europe (Radboud University Medical Center and the Donders Institute for Brain, Cognition and Behavior, Nijmegen, The Netherlands; Brain Center Rudolf Magnus, University Medical Center Utrecht, Utrecht, The Netherlands; King's College London, London, UK; Central Institute of Mental Health (CIMH), Mannheim, Germany). The measures used here were part of a larger test battery including questionnaires, neuropsychological testing, and MR scanning, as described in Naaijen et al (under review). Exclusion criteria for all participants were the presence of any contraindications for MRI, an $\mathrm{IQ}<70$, and the presence or history of neurological disorders. In addition, the ASD and OCD patients were not allowed to have comorbidity of the other disorder of interest. For the healthy comparison group, no DSM axis I disorders were allowed in any relatives up to two generations back. Ethical approval for the study was obtained for all sites separately. After description of the study, parents gave written informed consent, children aged 12 years or older gave written informed assent and children younger than 12 years gave oral informed assent.

\section{Phenotypic Information}

Diagnoses of ASD and OCD were informed by preassessment questionnaires including the Repetitive Behavior Scale (RBS) filled in by parents (Lam and Aman, 2007). Anxiety was measured by using a sub-scale of the Child Behavior Checklist (CBCL; Bordin et al, 2013). In addition, we used structured diagnostic interviews to confirm the diagnosis. The autism diagnostic interview revised (ADI-R; Lord et al, 1994), a structured developmental interview to assess the symptoms of ASD and classify ASD DSM-IV-TR diagnosis, was administered. The Children's Yale-Brown Obsessive Compulsive Scale (Scahill et al, 1997) was used as a severity scale for obsessions and compulsions for all OCD patients and whenever screening questions pointed to the presence of obsessions/compulsions in the other participant groups. In addition, all parents were interviewed using the 
structured Diagnostic Interview Schedule for Children (Shaffer et al, 2000), the Development and Well-being Assessment (Goodman et al, 2000), or the Kiddie Schedule for Affective Disorders, and Schizophrenia (Kaufman et al, 1997), dependent on site, to assess the presence of possible comorbidities, such as attention-deficit hyperactivity disorder (ADHD), a very common comorbidity in both ASD and OCD (Torres et al, 2016). This interview was also conducted in parents of control children to rule out the presence of DSM axis I diagnoses.

Full-scale IQ was estimated from four subtests (Vocabulary, Similarities, Block design, and Picture completion) of the Wechsler Intelligence Scale for Children (Wechsler, 2002). Medication information was collected via parental report.

\section{MR Acquisitions}

All scans were acquired on $3 \mathrm{~T}$ scanners (see Supplementary Table 1 for scanner information across sites). The scanning protocol included a structural T1-weighted MRI scan for the localization of the spectroscopy voxels for each participant and the two spectroscopy sequences. The structural T1-weighted scanning sequence was based on the ADNI GO protocols (Jack et al, 2008) and were matched as closely as possible across the different scanning sites (see Supplementary Table 1 for the T1-weighted scan parameters). Proton spectra were acquired using a point resolved spectroscopy (PRESS) sequence, similar across sites, with the chemically selective suppression (CHESS) water suppression technique (Haase et al, 1985). One $8 \mathrm{~cm}^{3}$ voxel $(2 \times 2 \times 2 \mathrm{~cm})$ was placed on the midline pregenual ACC anterior to and slightly superior of the genu of the corpus callosum, and the other was placed in the left dorsal striatum covering caudate and putamen $(\mathrm{TR}=3000 \mathrm{~ms}, \mathrm{TE}=30 \mathrm{~ms}$, number of averages $=96$, bandwidth $=5 \mathrm{kHz}$, number of points $=4096$ ). Additional unsuppressed water reference spectra (16 averages) were acquired. The voxel locations were adjusted to maximize their grey matter (GM) content (see Figure 1 for locations of the voxels as set by the experimenters across the different sites and Figure 2 for an example spectrum). Both voxels were placed to cover a maximum amount of GM and a minimum amount of cerebrospinal fluid (CSF). T1-weighted images were used for voxel placement and tissue segmentation. On-site training was provided before this study to minimize across-site variability in voxel placement. In addition, phantom (General Electric MRS phantom) and so-called 'travellinghead' data from all sites were collected to assure spectral quality and reliability across sites. Supplementary Figure 4 shows the spectra for one of the travelling heads.

\section{MR Processing and Modelling}

Analysis of acquired spectra was conducted using the Linear Combination Model (LCModel), version V6.03-0I (Provencher, 2001). LC model fits a linear combination of model metabolite spectra to the in vivo spectrum. The analyses were restricted to spectra with linewidth (full width at half maximum; FWHM) $\leqslant 0.1$ p.p.m., Cramér-Rao lower bounds (CRLBs) for glutamate $\leqslant 20 \%$, signal-to-noise ratio $\geqslant 5$, and concentrations within 2 SDs from the mean.

Eddy current corrections were performed and waterreferenced metabolite concentrations were automatically calculated in institutional units (i.u.; Gasparovic et al, 2006). I.u. are presented, as no $T_{1}$ and $T_{2}$ relaxation time corrections of the metabolite concentrations were performed. The $\mathrm{T}_{2}$ of tissue water was corrected for by assuming the signal had decayed by $30 \%$ at the echo time. In addition, there are other scanner-dependent factors that can affect the absolute scaling (eg, details of coil combination and differences in the radiofrequency pulse shapes, flip angles, and bandwidths) such that metabolite concentrations measured in i.u. are preferred over attempting to scale to absolute concentrations in $\mathrm{mM}$.

The unified segmentation procedure within the VBM8 toolbox of SPM8 (Statistical Parametric Mapping release 8, London, UK) was used to process the T1 images and generate GM, white matter (WM), and CSF probability maps in conjunction with non-linear transformations to the MNI 152 template space. Spectroscopy voxels were mapped onto these probability maps as well as onto the MNI 152 template, to
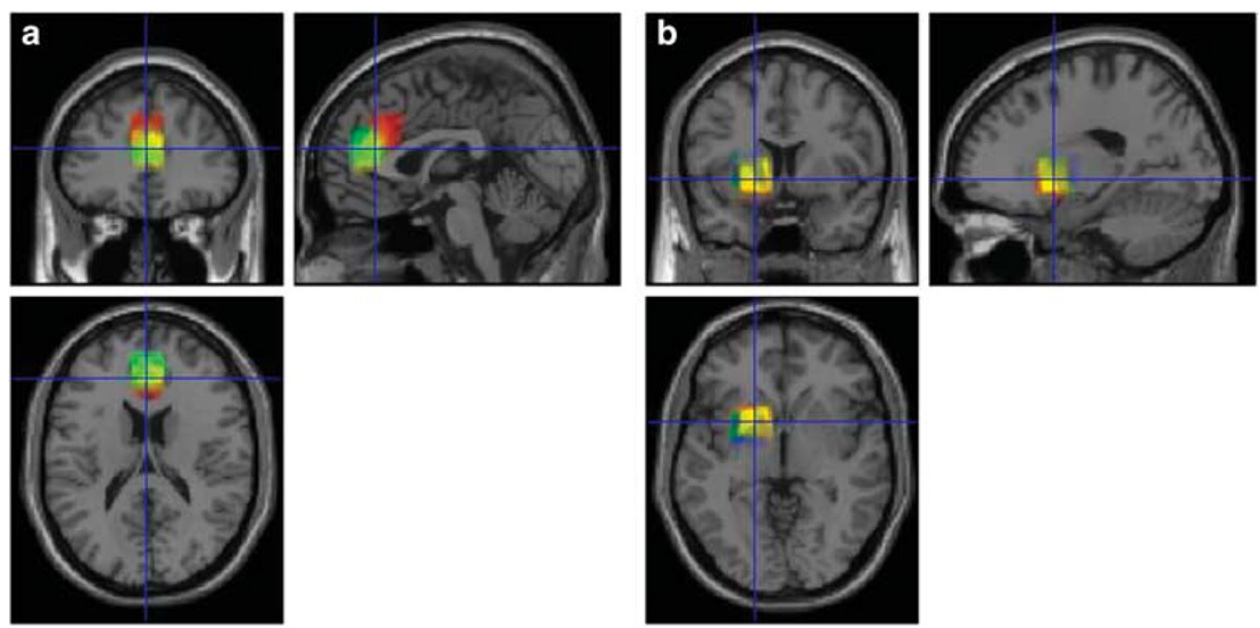

Figure I Superposition of all individual midline ACC (a) and left dorsal striatum voxels (b) on the MNII 52 template from London (blue), Mannheim (red), Nijmegen (yellow), and Utrecht (green). Note the consistent placement across the different sites and experimenters, as indicated by the large overlap and narrow spatial confinement of the colors. Individual positions of voxels per site can be found in Supplementary Figure SI. 
ACC
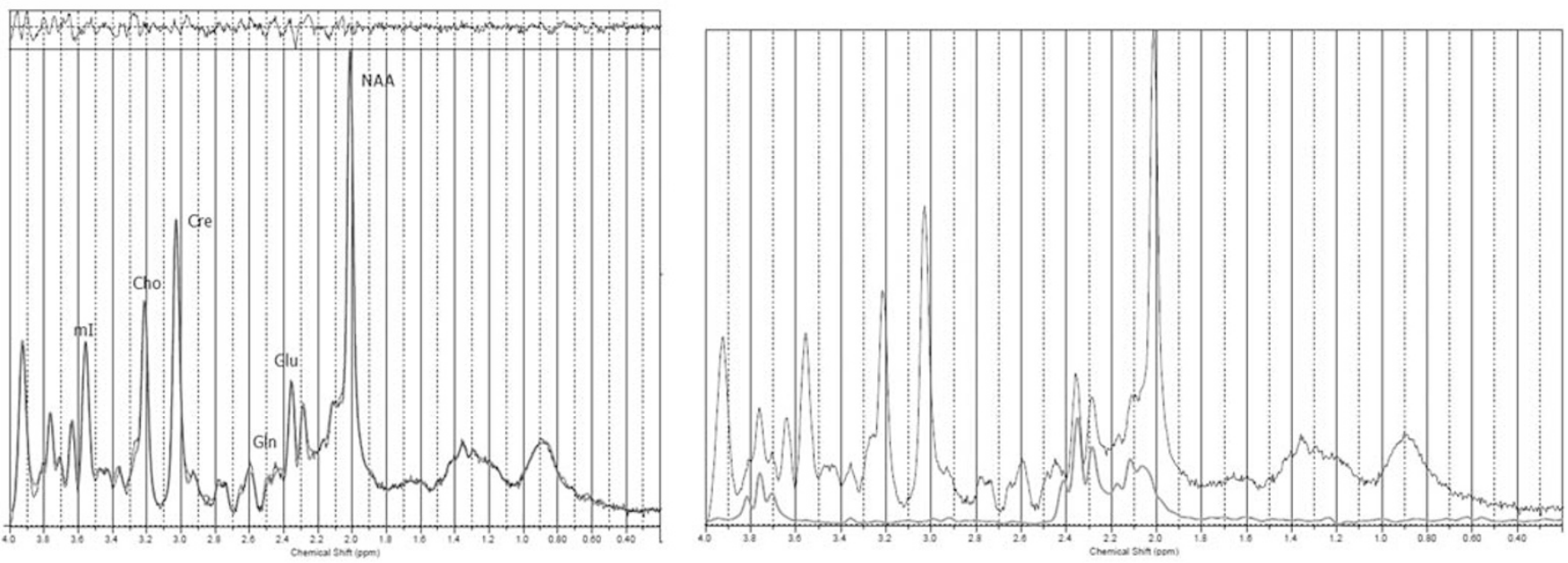

Striatum
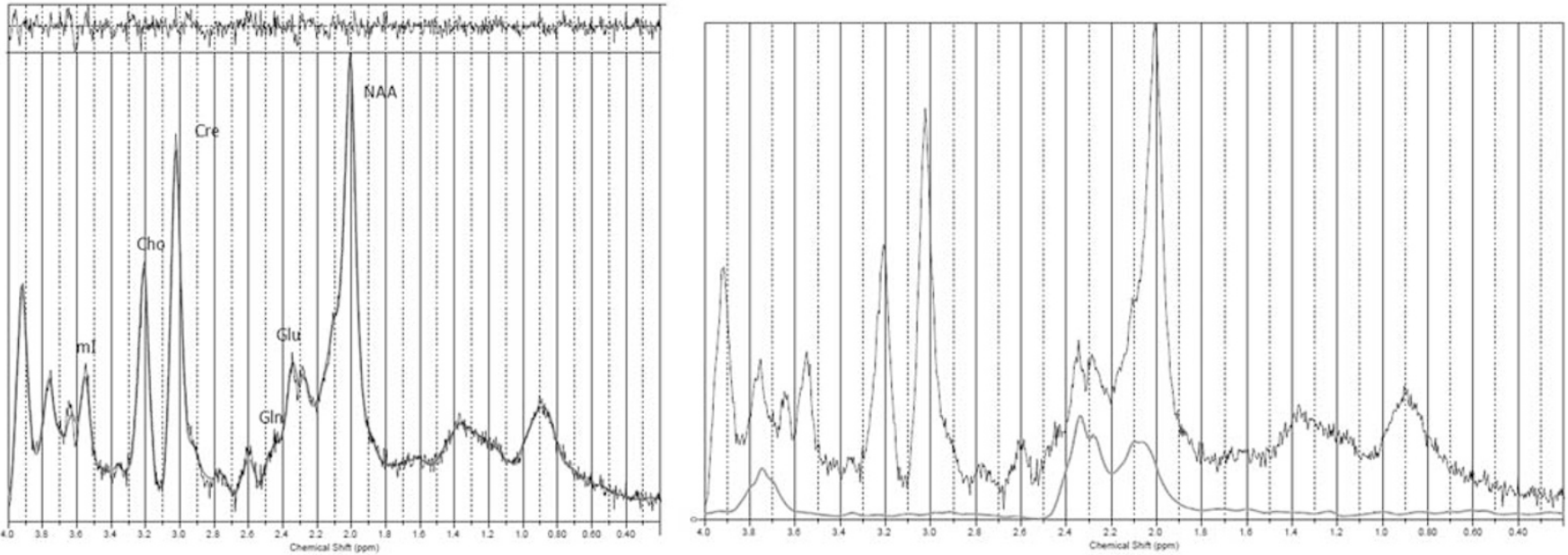

Figure 2 Representative example spectra of a 3 T proton magnetic resonance spectroscopy (IH-MRS) Linear Combination Model (LCModel) spectral fit in the ACC and the striatum, with a visualization of the fits for glutamate only. The thin black line represents the frequency-domain data, the red line is the LCModel fit. In the top panel, the residuals are plotted (the data minus the fit). A full color version of this figure is available at the Neuropsychopharmacology journal online.

provide the partial volume of GM, WM, and CSF within each spectroscopy voxel $\left(f_{\mathrm{GM}}, f_{\mathrm{WM}}\right.$, and $\left.f_{\mathrm{CSF}}\right)$, and to allow for group comparisons of the anatomical placement of the spectroscopy voxels. In addition, the amount of water in GM, WM, and CSF in each voxel was taken into account. To correct for differing amounts of water in each tissue and partial volume confounds (possible group differences in proportions of GM, WM, and CSF in the voxels), we corrected individual absolute metabolite concentrations with the following formula:

$$
\begin{aligned}
\text { Metabolite }_{\text {corrected }} & = \\
\text { Metabolite }_{\text {Raw }} & \times\left(\frac{43300 \times f_{\mathrm{GM}}+35880 \times f_{\mathrm{WM}}+55556 \times f_{\mathrm{CSF}}}{35880}\right) \\
& \times\left(\frac{1}{1-f_{\mathrm{CSF}}}\right)
\end{aligned}
$$

where 43300,35880 , and 55556 are the water concentrations in $\mathrm{mM}$ for GM, WM, and CSF, respectively. This includes a correction for the fraction of the voxel occupied by CSF, along with corrections for the water concentration in each of the tissue types. The factor 35880 in the denominator is included, as the initial LCModel analysis was carried out under the assumption of a pure WM voxel (LC model manual, Provencher, 2001).

\section{Statistical Analysis}

All statistical analyses were performed with SPSS release 21 (SPSS, Chicago, Illinois). First, demographic information was compared across groups. Sex was tested with Pearson's $\chi^{2}$-test. Group differences in continuous measures (age, IQ, and RBS score) were assessed with one-way analyses of variance (ANOVAs) if assumptions of homogeneity of variance and normality of distributions were met $(p>0.05$ in Levene's test of homogeneity of variance and ShapiroWilk normality test). If these assumptions were violated a non-parametric Kruskal-Wallis rank sum test (for three groups) or a Mann-Whitney $U$-test (for two groups) was used instead.

Glutamate levels were normalized using Blom transformations (also called rank-based inverse normal transformations; Conover and Iman, 1981) because of their non-normal 
Table I Demographic Characteristics (Based on the Group for the ACC Analysis)

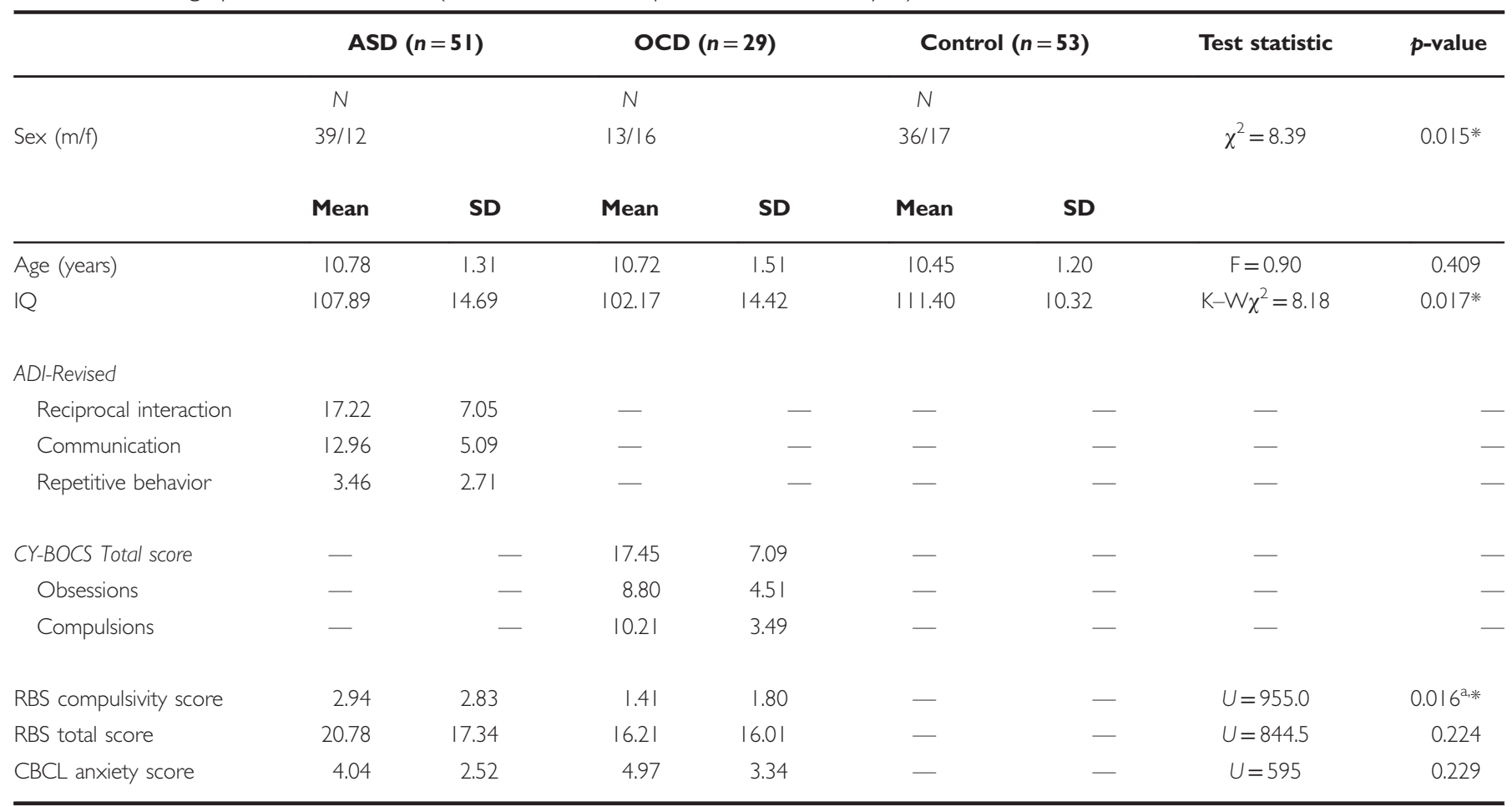

Abbreviations: ADI, Autism Diagnostic Interview (Lord et al, 1994); ASD, autism spectrum disorder; CBCL; Child Behavior Checklist (Bordin et al, 20I3); CY-BOCS, children's Yale-Brown Obsessive Compulsive scale (Scahill et al, 1997); m/f, male/female; OCD, obsessive compulsive disorder, RBS, Repetitive Behavior Scale (22); SD, standard deviation.

aDifference between the two diagnostic groups only.

*Significant at $p<0.05$.

distribution that allowed the use of subsequent parametric testing. Group differences in these glutamate levels were analyzed using an ANCOVA with scanner site (including major hardware upgrades on scanners across sites), medication use, sex, and age as covariates. Covariates were removed from analysis, if they did not significantly contribute to the explained variance of the model. Subsequently, contrast analyses were performed to compare the two disorder groups with controls and with each other. A $p$-value of $<0.025$ after correction for multiple comparisons (two voxels of interest) was considered statistically significant. We used the same analysis for Glx in the Supplementary Material.

For metabolites that were significantly different across the groups per voxel, post hoc correlation analyses with RBS compulsivity, RBS total scores, and CBCL anxiety scores were performed using non-parametric correlation analyses (Spearman's rho) due to the nonlinear relationship between glutamate levels and these behavioral measures. The correlation analyses were performed for the disorder groups only, as compulsivity scores were $\leqslant 1$ for the controls.

\section{RESULTS}

\section{Demographics}

MR spectra were acquired for 143 of the original 163 participants. Thirteen participants with ASD, two participants with OCD, and five healthy controls were excluded due to excessive movement, anxiety in the scanner, or not meeting full diagnostic criteria after inspection of the ADI-R scores. Due to spectral or segmentation quality concerns, 10 additional spectra were excluded from ACC analysis $(n=133)$ and 29 from striatal analysis $(n=114)$. Table 1 provides a summary of the demographic information. Sex and IQ differed between the three groups. Supplementary Table 2 provides demographic information across the four sites. The exclusion of more participants from the striatal analyses did not influence demographic distributions in the group, regarding sex $\left(\chi^{2}=6.88, p=0.032\right)$, age $(\mathrm{F}=0.60$, $p=0.55$ ), RBS-scores (compulsivity: $U=695.5, p=0.012$; total: $U=621, p=0.151)$ and CBCL-anxiety $(U=430$, $p=0.34)$. However, the difference in IQ became trend-level in this group $\left(\mathrm{K}-\mathrm{W} \chi^{2}=5.42, p=0.07\right)$.

Children both on and off medication were included. Within the ASD group, three children used stimulants, two used stimulants and antipsychotics, two stimulants and atomoxetine, one antipsychotics, and one child used naltrexone. From the children with OCD, five used antipsychotics, one used antipsychotics and atomoxetine, three used stimulants, and two were on anti-depressants. Participants were asked to abstain from medication $48 \mathrm{~h}$ before scanning.

Several comorbidities were present across disorder groups, with ASD participants showing comorbid ADHD $(n=13)$, ODD $(n=7), \mathrm{CD}(n=1)$, and tic disorder $(n=5)$. In the OCD group, the following comorbidities were present: ADHD $(n=10)$, ODD $(n=2), \mathrm{CD}(n=3)$, tic disorder $(n=3)$, social anxiety disorder $(n=1)$, generalized anxiety disorder $(n=4)$, major depression $(n=1)$, and dysthymia $(n=1)$. 


\section{Spectral Quality Assurance}

Voxel composition did not differ between ASD, OCD, and controls in either ACC or striatum (Table 2), supporting that our results were not confounded by partial volume effects. To confirm that spectral quality did not differ between groups, we compared the CRLB-estimated SDs in both voxels using a one-way ANOVA across the three groups. CRLBs did not differ between groups in ACC $\left(\mathrm{F}_{(2,130)}=0.41\right.$, $p=0.67)$ or striatum $\left(\mathrm{F}_{(2,111)}=0.02, p=0.99\right)$, confirming that possible differences in glutamate levels were not due to differences in CRLBs (Kreis, 2015). Also, we did not find any differences in FWHM (ACC: $\mathrm{F}_{(2,130)}=0.52, p=0.60$; striatum: $\left.\mathrm{F}_{(2,111)}=0.25, p=0.78\right)$ or SNR $\left(\right.$ ACC: $\mathrm{F}_{(2,130)}=1.54$, $p=0.22$; striatum: $\left.\mathrm{F}_{(2,111)}=0.57, p=0.57\right)$. Glutamate closely interacts with glutamine and it is suggested that glutamine concentrations may be additionally informative about possible glutamate dysfunction (Bak et al, 2006). LC model fits for glutamine were poor, showing high CRLBs. We therefore did not include glutamine or the combined Glx signal in analysis.

As this was the first multi-center MRS study investigating ASD and OCD together, we also investigated whether any site differences, present because of the different scanners, affected glutamate levels differently across the three groups. The interaction term of site and diagnosis did not affect glutamate levels in ACC or striatum (both $p$-values $>0.1$ ). Also, voxel tissue composition did not differ between groups across sites (all $p$-values $>0.1$ ). The Supplementary Material provides additional information about voxel placement across sites and site/diagnosis interaction plots in terms of partial volumes. It provides additional visual representation of the spectra across the four sites for a travelling head.

\section{Group Comparisons}

A group difference was found in corrected glutamate levels in ACC, while including sex, medication use, and scanner site as covariates $\left(\mathrm{F}_{(2,127)}=4.12, p=0.019\right.$; see Table 2 and Figure 3). Age did not influence the ANCOVA model and was therefore excluded. Although there were differences in sex between groups (with more males in the ASD and control group compared with the OCD group), they did not significantly influence the Glu levels $\left(\mathrm{F}_{(1,127)}=1,92, p=0.17\right)$. Post hoc between-group contrasts were performed to obtain a more detailed understanding of the nature of the effect. Indeed, these tests showed higher Glu levels in ACC of the two disorder groups (combined) compared with controls $(t=2.85, p=0.007)$, whereas the two disorder groups did not differ from each other $(t=0.29, p=0.77)$. Separately, both ASD $(t=2.52, p=0.014)$ and $\operatorname{OCD}(t=2.36, p=0.02)$ showed increased Glu levels in the ACC compared with controls as well, demonstrating that it was not driven by either one of the disorders. We subsequently compared Glu levels in a restricted set of patients with ASD and OCD with no prior or current use of any medication (ASD, $n=39$; OCD, $n=20$ ) and separately for patients without any comorbidities (ASD, $n=33$; OCD, $n=15$ ). Glu levels also differed between the groups in these more limited samples of medication-free participants $\left(\mathrm{F}_{(2,107)}=4.13, p=0.019\right)$ and comorbidity-free participants $\left(\mathrm{F}_{(2,94)}=4.65, p=0.012\right)$; post hoc across group tests revealed the same results as in the larger sample. Dimensional analysis showed a positive correlation between Glu levels in the ACC and compulsivity as measured with the RBS ( $r h o=0.24, p=0.03$; Figure 4). The correlation was not present when we investigated ASD and OCD separately (both $p$-values $>0.05$ ), possibly due to the smaller samples. No significant correlation was observed between Glu levels in the ACC and more general repetitive behavior as measured with the RBS total (excluding the

Table 2 Voxel Composition (Partial Volumes) and Glutamate Concentrations

\begin{tabular}{|c|c|c|c|c|c|c|c|c|}
\hline & \multicolumn{2}{|c|}{ ASD } & \multicolumn{2}{|c|}{ OCD } & \multicolumn{2}{|c|}{ Control } & \multirow[t]{2}{*}{ F statistic } & \multirow[t]{2}{*}{$p$-value } \\
\hline & Mean & SE & Mean & SE & Mean & SE & & \\
\hline \multicolumn{9}{|l|}{$A C C$} \\
\hline$\%$ Grey matter & 67.8 & 1.4 & 67.8 & 1.9 & 67.0 & 1.4 & 0.98 & 0.91 \\
\hline$\%$ White matter & 11.6 & 0.5 & 11.3 & 0.7 & 12.5 & 0.5 & 1.17 & 0.32 \\
\hline Normalized Glua & 0.17 & 0.13 & 0.23 & 0.17 & -0.29 & 0.13 & 4.11 & $0.019 *$ \\
\hline \multicolumn{9}{|l|}{ Striatum } \\
\hline \% Grey matter & 56.0 & 1.4 & 57.3 & 1.8 & 58.1 & 1.3 & 0.58 & 0.56 \\
\hline
\end{tabular}

Abbreviations: ACC, anterior cingulate cortex; ASD, autism spectrum disorder; CSF, cerebrospinal fluid; $O C D$, obsessive compulsive disorder; Glu, glutamate. abased on the estimated marginal means (adjusted for covariate use).

*Significant at $p<0.05$. 


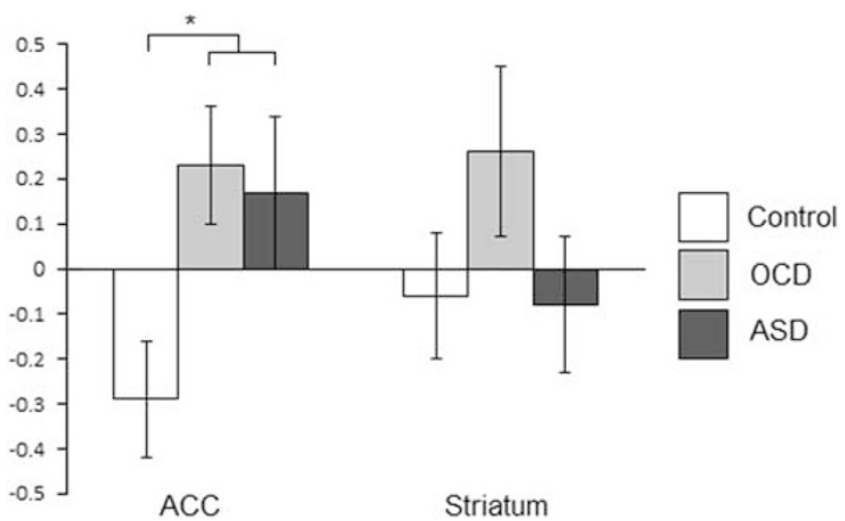

Figure 3 Glutamate in the anterior cingulate cortex (ACC; left) and the dorsal striatum (right) across the three groups. Glutamate is plotted as a normalized value (using Blom transformation). The asterisk indicates the significant difference between the disorder groups and controls. (ASD, autism spectrum disorder; OCD, obsessive compulsive disorder.)

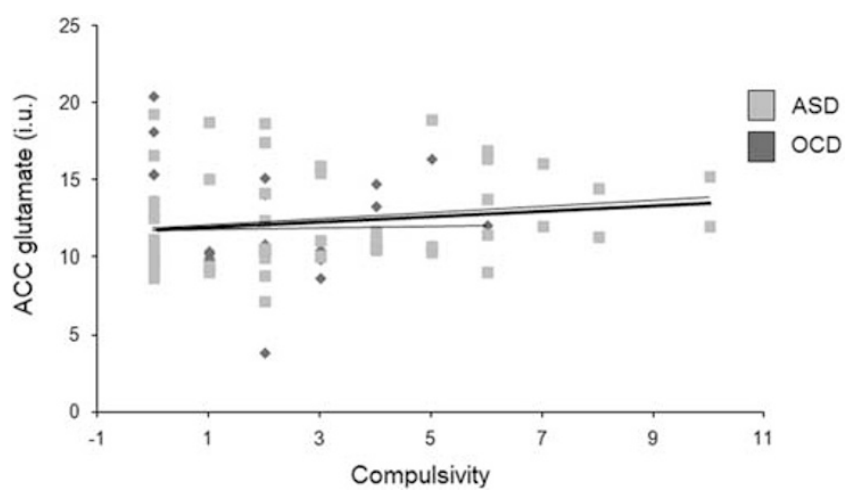

Figure 4 Relation between anterior cingulate cortex (ACC) glutamate levels and compulsivity scores for participants with autism spectrum disorder (ASD; red) and obsessive compulsive disorder (OCD; green), with fit lines per group. The solid black line represents the total fit line. A full color version of this figure is available at the Neuropsychopharmacology journal online.

compulsivity scale) (rho $=0.08, p=0.49)$. Also no correlation was found with anxiety ( $r h o=-0.12, p=0.30)$. We additionally calculated the difference between the correlations (Lee and Preacher, 2013). The correlation between compulsivity and glutamate and general repetitive behavior and glutamate differed significantly from each other $(p=0.03)$ as did the compulsivity and glutamate and anxiety and glutamate $(p=0.005)$.

No group differences in absolute Glu levels were found in the striatum $\left(\mathrm{F}_{(2,108)}=1.19, p=0.31\right.$; Figure 3$)$.

We additionally found group differences in ACC Glx concentrations, but not in striatum Glx concentrations (Supplementary Material).

\section{DISCUSSION}

This is the first study to assess glutamate levels in the frontostriatal circuit in two compulsivity disorders, ASD and OCD, and in healthy controls. We conducted both categorical analyses contrasting diagnostic groups and dimensional analyses using a continuous measure of compulsivity.
We observed glutamate levels in the ACC to be significantly higher in children with compulsivity disorders (ASD and OCD) compared with controls. No difference was found between the two disorder groups. ACC Glx levels were increased in children with compulsivity disorders as well. Several previous studies investigating children with ASD have found increased Glu or Glx levels in ACC (Bejjani et al, 2012; Hassan et al, 2013; Joshi et al, 2012). Adults with ASD, on the other hand, tend to show decreased levels of glutamatergic compounds in prefrontal brain regions (Naaijen et al, 2015). It thus seems that glutamatergic over-activity in the ACC may be specific for children with ASD. This over-activity may not be limited to the ACC, as previous studies also reported increased glutamate levels within the amygdala-hippocampal complex (Page et al, 2006) as well as in the cerebellum and striatum (Hassan et al, 2013). The latter was not confirmed in the current study. The pattern of higher-than-normal glutamate metabolite levels in early stages of development and lowerthan-normal levels in adulthood may point to excitotoxicity in youth attenuating glutamate signalling in adulthood (Joshi et al, 2012). However, also in controls, metabolite levels are known to change with age (Horska et al, 2002).

In OCD, the findings have been less robust so far, with studies showing increased Glx levels in the caudate (Gnanavel et al, 2014) and orbitofrontal cortex (Whiteside et al, 2006,) but also decreased levels across different brain regions, including ACC (Rosenberg et al, 2004; Yücel et al, 2008). Most studies to date have been performed in adults and most were unable to find any differences between patients and controls (Brennan et al, 2013; Naaijen et al, 2015). Here we specifically focused on an important agerange of 8-13 years within neurodevelopment. Our data thus fill an important void in current research, especially given the knowledge about age-specific effects in the overlapping disorder ASD (Naaijen et al, 2015).

The most important result of the current study is that there was no difference in glutamate levels in the ACC between the two disorders, whereas both disorders differed significantly from controls. This similarity across the two disorders might be due to common underlying mechanisms of compulsivity. This is also consistent with the result of the correlation analysis, where increased compulsivity was associated with increased ACC glutamate levels, although the correlation was quite small. This correlation was not found with the total score on the RBS or with anxiety, which suggests that ACC glutamate is more specifically involved in compulsive behavior and not in anxiety and other aspects related to repetitive behavior, such as self-harm, resistance to change, and restricted interests. This cross-disorder analysis of the dimensional measure provides more insights into the underlying mechanism of the compulsivity seen in ASD and OCD, in concordance with the approach of the Research Domain Criteria (Cuthbert, 2014).

Increased Glu levels in ACC suggest a hyper-glutamatergic brain state, as previously has been hypothesized in ASD (Fatemi, 2008). This is supported by genetic studies pointing to involvement of GRIN2B, a gene encoding the glutamate NMDA receptor $2 \mathrm{~B}$, in both ASD and OCD (eg, Alonso et al, 2012). Also, the proteins GAD65kDA and GAD67kDA, responsible for converting glutamate to GABA, have been shown to be reduced in ASD, which may result in elevated glutamate levels by reducing inhibitory GABAergic tone 
(Pizzarelli and Cherubini, 2011). Future studies should incorporate the influence of glutamate genes on the brain glutamate signal and investigate the influence of glutamatergic medication on brain glutamate levels. We did not find any differences in striatal glutamate, which was not due to differences in quality across the disorders. Striatal spectra, however, are very hard to acquire (Hess et al, 2013) and we may have had reduced power to detect differences because of the lower spectra quality. However, the fronto-striatal deficits described in compulsivity disorders may also just be due more to deficits in self-control and regulation of compulsive behavior performed by the frontal areas and less to the more subcortical functions driving the compulsivity or the habitual behaviors (ie, Dalley et al, 2011 and Fineberg et al, 2010).

Strengths of the current study are the combination of a categorical (two disorder groups in one comparison) and a dimensional approach to investigate the relation between compulsivity and glutamate. There were, however, also some limitations. First, compulsivity is also often associated with dysfunctions of (glutamate in) the orbitofrontal cortex (Brennan et al, 2013; Naaijen et al, 2015), which we did not include in the current study. Pilot spectral acquisitions in this region were of low quality due to field inhomogeneities and lipid contamination.

Second, the OCD group was smaller than the ASD and control group due to recruitment difficulties. This resulted in less power and may have led to false negative results in the striatum, also findings in ACC only reached nominal significance. However, the continuous measure of compulsivity confirmed the link between compulsive behavior and ACC glutamate. Last, the groups were quite heterogeneous in terms of medication use and comorbidities. The group difference was retained, however, when we investigated only participants that did not use any medication.

In conclusion, the current study showed increased glutamate levels in the ACC of children with compulsivity disorders compared with controls and a relation between compulsivity severity and the ACC glutamate levels. Although there were effects of scanner sites on glutamatergic metabolites, these effects did not differ between or interact with diagnostic group status, showing the robustness of these findings across different sites and scanners. These results need replication in an independent sample but nevertheless provide insights into the involvement of glutamate in pediatric ASD and OCD, and the need for further exploration of glutamatergic interventions in these disorders.

\section{FUNDING AND DISCLOSURE}

The research leading to these results has received funding from the European Community's Seventh Framework Program (FP7/2007-2013) TACTICS under grant agreement no. 278948. This research was also supported from the Innovative Medicines Initiative Joint Undertaking under grant agreement number 115300 (EU-AIMS), resources of which are composed of financial contribution from the European Union's Seventh Framework Programme (FP7/2007 - 2013) and the European Federation of Pharmaceutical Industries and Associations (EFPIA) companies' in kind contribution. B Franke is supported by a Vici grant of the Netherlands Organization for Scientific Research (NWO, grant number (016-130-669). D Brandeis serves as an unpaid scientific advisor for an EU-funded Neurofeedback trial unrelated to the present work. T Banaschewski served in an advisory or consultancy role for Actelion, Hexal Pharma, Lilly, Medice, Novartis, Oxford outcomes, PCM scientific, Shire, and Viforpharma. He received conference support or speaker's fee by Medice, Novartis, and Shire. He is/has been involved in clinical trials conducted by Shire and Viforpharma. The present work is unrelated to the grants and relationships noted earlier. JC Glennon has acted as a consultant for Boehringer Ingelheim GmbH. B. Franke received an educational speaking fee from Merz. JK Buitelaar has been consultant to/member of advisory board of and/or speaker for Janssen Cilag BV, Eli Lilly, BristolMyer Squibb, Shering Plough, UCB, Shire, Novartis, and Servier. He is not an employee of any of these companies, nor a stock shareholder of any of these companies. He has no other financial or material support, including expert testimony, patents, and royalties. DJ Lythgoe has acted as a consultant for Ixico PLC. The remaining authors declare no conflict of interest.

\section{ACKNOWLEDGMENTS}

The TACTICS Consortium consists of Jan Buitelaar, Saskia de Ruiter, Jilly Naaijen, Sophie Akkermans, Maarten Mennes, Marcel Zwiers, Shahrzad Ilbegi, Leonie Hennissen, Jeffrey Glennon, Ilse van de Vondervoort, Katarzyna Kapusta, Natalia Bielczyk, Houshang Amiri, Martha Havenith, Barbara Franke, Geert Poelmans, Janita Bralten, Tom Heskes, Elena Sokolova, Perry Groot from Radboud University Medical Center Nijmegen, the Netherlands; Steven Williams, David Lythgoe, Muriel Bruchhage, Iulia Dud from Kings College London, United Kingdom; Ralf Dittmann, Tobias Banaschewski, Daniel Brandeis, Konstantin Mechler, Ruth Berg, Isabella Wolf, Alexander Häge, Sarah Hohmann, Regina Boecker, Matthias Ruff from Central Institute of Mental Health, University of Heidelber, Mannheim, Germany; Rick Dijkhuizen, Erwin Blezer, Kajo van der Marel, Pim Pullens, Wouter Mol, Annette van der Toorn, Willem Otte, Caroline van Heijningen, Sarah Durston, Vincent Mensen, Bob Oranje, René Mandl from University Medical Center Utrecht, Utrecht, the Netherlands; Daphna Joel from Tel Aviv University, Tel Aviv, Israel; John Cryan from University College Cork, Cork City, Ireland; Tracey Petryshen, David Pauls, Mai Saito from Massachusetts General Hospital, Boston, USA; Angelique Heckman from Genoway, Lyon, France; Sabine Bahn from University of Cambridge, Cambridge, United Kingdrom; Ameli Schwalber from Concentris, München, Germany; and Philippe Auby from Lundbeck, Valby, Denmark.

We gratefully acknowledge and thank all the participants for their enthusiastic involvement in the study. Data contributing to this research were presented as a scientific poster at the European College for Neuropsychopharmacology (ECNP) meeting 2015, Amsterdam, The Netherlands, and 2016, Vienna, Austria.

\section{REFERENCES}

Alexander GE, Delong MR, Strick PL (1986). Parallel organization of functionally segregated circuits linking basal ganglia and cortex. Annu Rev Neurosci 9: 357-381. 
Alonso P, Gratacós M, Segalàs C, Escaramís G, Real E, Bayés M et al (2012). Association between the NMDA glutamate receptor GRIN2B gene and obsessive-compulsive disorder. I Psychiatry Neurosci 37: 273-281.

American Psychiatric Association (2000). Diagnostic Criteria From $D S M-I V-T R$. American Psychiatric Association: Washington, DC, USA.

Anholt GE, Cath DC, van Oppen P, Eikelenboom M, Smit JH, van Megen $\mathrm{H}$ et al (2010). Autism and ADHD symptoms in patients with OCD: are they associated with specific OC symptom dimensions or OC symptom severity? J Autism Dev Disord 40: 580-589.

Bak LK, Schousboe A, Waagepetersen HS (2006). The glutamate/ GABA-glutamine cycle: aspects of transport, neurotransmitter homeostasis and ammonia transfer. J Neurochem 98: 641-653.

Bejjani A, O’Neill J, Kim Ja, Frew AJ, Yee VW, Ly R et al (2012). Elevated glutamatergic compounds in pregenual anterior cingulate in pediatric autism spectrum disorder demonstrated by $1 \mathrm{H}$ MRS and 1H MRSI. PLoS One 7: e38786.

Bordin IA, Rocha MM, Paula CS, Teixeira MC, Achenbach TM, Rescorla LA et al (2013). Child Behavior Checklist (CBCL), Youth Self-Report (YSR) and Teacher's Report Form(TRF): an overview of the development of the original and Brazilian versions. Cad Saude Publica 29: 13-28.

Brennan BP, Rauch SL, Jensen JE Jr, Pope HG (2013). A critical review of magnetic resonance spectroscopy studies of obsessivecompulsive disorder. Biol Psychiatry 73: 24-31.

Chamberlain SR, Menzies L (2009). Endophenotypes of obsessivecompulsive disorder: rationale, evidence and future potential. Expert Rev Neurother 9: 1133-1146.

Conover WJ, Iman RL (1981). Rank transformations as a bridge between parametric and nonparametric statistics. Am Stat 35: 124-129.

Cuthbert BN (2014). The RDoC framework: facilitating transition from ICD/DSM to dimensional approaches that integrate neuroscience and psychopathology. World Psychiatry 13: 28-35.

Dalley JW, Everitt BJ, Robbins TW (2011). Impulsivity, compulsivity, and top-down cognitive control. Neuron 69: 680-694.

Fatemi SH (2008). The hyperglutamatergic hypothesis of autism. Prog Neuropsychopharmacol Biol Psychiatry 32: 911.

Fineberg Na, Potenza MN, Chamberlain SR, Berlin Ha, Menzies L, Bechara A et al (2010). Probing compulsive and impulsive behaviors, from animal models to endophenotypes: a narrative review. Neuropsychopharmacology 35: 591-604.

Fuccillo MV (2016). Striatal circuits as a common node for autism pathophysiology. Front Neurosci 10: 27.

Gasparovic C, Song T, Devier D, Bockholt HJ, Caprihan A, Mullins PG et al (2006). Use of tissue water as a concentration reference for proton spectroscopic imaging. Magn Reson Med 55: 1219-1226.

Gnanavel S, Sharan P, Khandelwal S, Sharma U, Jagannathan NR (2014). Neurochemicals measured by (1)H-MR spectroscopy: putative vulnerability biomarkers for obsessive compulsive disorder. MAGMA 27: 407-417.

Goodman R, Ford T, Richards H, Gatward R, Meltzer H (2000). The Development and Well-Being Assessment: description and initial validation of an integrated assessment of child and adolescent psychopathology. J Child Psychol Psychiatry 41: 645-655.

Haase A, Frahm J, Hänicke W, Matthaei D (1985). 1H NMR chemical shift selective (CHESS) imaging. Phys Med Biol 30: 341-344.

Hassan TH, Abdelrahman HM, Abdel NR, El-masry NM, Hashim HM, El-gerby KM et al (2013). Research in Autism spectrum disorders blood and brain glutamate levels in children with autistic disorder. Res Autism Spectr Disord 7: 541-548.

Hess AT, Van Der Kouwe AJW, Mbugua KK, Laughton B, Meintjes EM (2013). Quality of 186 child brain spectra using motion and b0 shim navigated single voxel spectroscopy. J Magn Reson Imaging 40: 958-965.

Horska A, Kaufmann WE, Brant LJ, Naidu S, Harris JC, Barker PB (2002). In vivo quantitative proton MRSI study of brain development from childhood to adolescence. J Magn Reson Imaging 15: 137-143.

Hyder F, Fulbright RK, Shulman RG, Rothman DL (2013). Glutamatergic function in the resting awake human brain is supported by uniformly high oxidative energy. J Cereb Blood Flow Metab 33: 339-347.

Jack CR, Bernstein MA, Fox NC, Thompson P, Alexander G, Harvey D et al (2008). The Alzheimer's Disease Neuroimaging Initiative (ADNI): MRI methods. J Magn Reson Imaging 27: 685-691.

Joshi G, Biederman J, Wozniak J, Goldin RL, Crowley D, Furtak S (2012). Magnetic resonance spectroscopy study of the glutamatergic system in adolescent males with high-functioning autistic disorder: a pilot study at 4T. Eur Arch Psychiatry Clin Neurosci 263: 379-384.

Kaufman J, Birmaher B, Brent D, Rao U, Flynn C, Moreci P et al (1997). Schedule for affective Disorders and Schizophrenia for School-Age Children Present and Lifetime version (K-SADS-PL): initial reliability and validity data. J Am Acad Child Adolesc Psychiatry 36: 980-988.

Klumpp H, Ho SS, Taylor SF, Phan KL, Abelson JL, Liberzon I (2011). Trait anxiety modulates anterior cingulate activation to threat interference. Depress Anxiety 28: 194-201.

Kreis R (2015). The trouble with quality filtering based on relative Cramèr-Rao lower bounds. Magn Reson Med 75: 15-18.

Lam KS, Aman MG (2007). The Repetitive Behavior Scale-Revised: independent validation in individuals with autism spectrum disorders. J Autism Dev Disord 37: 855-866.

Lee IA, Preacher KJ (2013). Calculation for the test of the difference between two dependent correlations with one variable in common. (Comput Software). Available at http:/quantpsy.org/ corrtest/corrtest2.htm.

Lord C, Rutter M, Couteur AL (1994). Autism diagnostic interviewrevised: A revised version of a diagnostic interview for caregivers of individuals with possible pervasive developmental disorders. J Autism Dev Disord 24: 659-685.

Meier SM, Petersen L, Schendel DE, Mattheisen M, Mortensen PB, Mors O (2015). Obsessive-compulsive disorder and autism spectrum disorders: longitudinal and offspring risk. PLoS One 10: e0141703.

Modi S, Rana P, Kaur P, Rani N, Khushu S (2014). Glutamate level in anterior cingulate predicts anxiety in healthy humans: a magnetic resonance spectroscopy study. Psychiatry Res 224: 34-41.

Naaijen J, Lythgoe DJ, Amiri H, Buitelaar JK, Glennon JC (2015). Fronto-striatal glutamatergic compounds in compulsive and impulsive syndromes: a review of magnetic resonance spectroscopy studies. Neurosci Biobehav Rev 52: 74-88.

Page LA, Daly E, Schmitz N, Simmons A, Toal F, Deeley Q et al (2006). In vivo $1 \mathrm{H}$-magnetic resonance spectroscopy study of amygdala-hippocampal and parietal regions in autism. Am J Psychiatry 163: 2189-2192.

Pittenger C, Bloch MH, Williams K (2011). Glutamate abnormalities in obsessive compulsive disorder: neurobiology, pathophysiology, and treatment. Pharmacol Ther 132: 314-332.

Pizzarelli R, Cherubini E (2011). Alterations of GABAergic signaling in autism spectrum disorders. Neural Plast 2011: 297153.

Provencher SW (2001). Automatic quantitation of localized in vivo 1H spectra with LCModel. NMR Biomed 14: 260-264.

Robbins TW, Gillan CM, Smith DG, de Wit S, Ersche KD (2012). Neurocognitive endophenotypes of impulsivity and compulsivity: towards dimensional psychiatry. Trends Cogn Sci 16: 81-91.

Rosenberg DR, MacMaster FP, Keshavan MS, Fitzgerald KD, Stewart CM, Moore GJ (2000). Decrease in caudate glutamatergic concentrations in pediatric obsessive-compulsive disorder 
patients taking paroxetine. J Am Acad Child Adolesc Psychiatry 39: 1096-1103.

Rosenberg DR, Mirza Y, Russell A, Tang J, Smith JM, Banerjee SP et al (2004). Reduced anterior cingulate glutamatergic concentrations in childhood OCD and major depression versus healthy controls. J Am Acad Child Adolesc Psychiatry 43: 1146-1153.

Ruzzano L, Borsboom D, Geurts HM (2015). Repetitive behaviors in autism and obsessive-compulsive disorder: new perspectives from a network analysis. J Autism Dev Disord 45: 192-202.

Scahill L, Riddle MA, McSwiggin-Hardin M, Ort SI, King RA, Goodman WK et al (1997). Children's Yale-Brown Obsessive Compulsive Scale: reliability and validity. J Am Acad Child Adolesc Psychiatry 36: 844-852.

Shaffer D, Fisher P, Lucas CP, Dulcan MK, Schwab-Stone ME (2000). NIMH Diagnostic Interview Schedule for Children Version IV (NIMH DISC-IV): description, differences from previous versions, and reliability of some common diagnoses. J Am Acad Child Adolesc Psychiatry 39: 28-38.

Somerville LH, Casey BJ (2010). Developmental neurobiology of cognitive control and motivational systems. Curr Opin Neurobiol 20: $271-277$.
Thakkar KN, Polli FE, Joseph RM, Tuch DS, Hadjikhani N, Barton JJ et al (2008). Response monitoring, repetitive behaviour and anterior cingulate abnormalities in autism spectrum disorders (ASD). Brain 131: 2464-2478.

Torres AR, Fontenelle LF, Shavitt RG, Ferrão YA, Do Rosário MC, Storch EA et al (2016). Comorbidity variation in patients with obsessive-compulsive disorder according to symptom dimensions: results from a large multicentre clinical sample. J Affect Disord 190: 508-516.

Voon V, Derbyshire K, Rück C, Irvine MA, Worbe Y, Enander J et al (2014). Disorders of compulsivity: a common bias towards learning habits. Mol Psychiatry 20: 345-352.

Wechsler D (2002). WISC-III Handleiding. The Psychological Corporation: London.

Whiteside SP, Port JD, Deacon BJ, Abramowitz JS (2006). A magnetic resonance spectroscopy investigation of obsessivecompulsive disorder and anxiety. Psychiatry Res 146: 137-147.

Yücel M, Wood SJ, Wellard RM, Harrison BJ, Fornito A, Pujol J et al (2008). Anterior cingulate glutamate-glutamine levels predict symptom severity in women with obsessivecompulsive disorder. Aust NZ J Psychiatry 42: 467-477.

Supplementary Information accompanies the paper on the Neuropsychopharmacology website (http://www.nature.com/npp) 